\title{
Some aspects of Harmonic Numbers which divide the sum of its Positive divisors.
}

\author{
Pranjal Rajkhowa ${ }^{1}$, Hemen Bharali ${ }^{2}$ \\ ${ }^{I}$ (Department of Mathematics), Gauhati University. India \\ ${ }^{2}$ (Department of Basic Sciences),Don Bosco College of Engineering and Technology ,Guwahati ,India
}

\begin{abstract}
In this paper some particular harmonic numbers $n$, where $n$ divides $\sigma(n)$ completely have been taken. Three types of numbers prime factorised as say, $p^{m} q, 2^{k} p^{m} q, 2^{k} p_{1} p_{2} \ldots \ldots p_{m}$ have been discussed and some propositions have been developed to understand the properties of these type of numbers. It has been observed that harmonic numbers $n$ having g.c.d. $(n, \sigma(n))=n$ of the form $p q, p^{2} q, p^{m} q$ does not exist if $p, q$ both of them are odd primes. Further some useful properties of the harmonic numbers of the form $2^{k} p^{m} q$, for some values of $m$ have been discussed with the help of some propositions. After that an algorithm has been proposed to get the numbers of the form $2^{k} p_{1} p_{2} \ldots \ldots p_{m}$, where $n$ divides $\sigma(n)$ completely.
\end{abstract}

Keywords: Harmonic number, Mersene prime, Ore's conjecture, Perfect number

\section{Introduction}

In 1948 Ore [9] introduced the concept of Harmonic numbers, and these numbers were named as Ore's harmonic numbers(some 15 years later) by Pomerance[10].In general, the harmonic mean of positive numbers $a_{1}, a_{2}, \ldots \ldots ., a_{k}$ is defined by

$$
\left(\sum_{i=1}^{k} \frac{1}{a_{i}}\right)^{-1}
$$

A positive integer $\mathrm{n}$ is said to be harmonic if the harmonic mean of its positive divisors $H(n)=\frac{n \tau(n)}{\sigma(n)}$ is an integer, where $\tau(n)$ denotes the number of positive divisors and $\sigma(\mathrm{n})$ denotes the sum of the positive divisors of $\mathrm{n}$. We call 1 the trivial harmonic number. A list of harmonic numbers less than $2.10^{9}$ have been given by Cohen [2]. This have been extended by Goto and Okeya [5] which is up to $10^{14}$. If $\mathrm{n}$ is a harmonic divisor number then there are two possibilities (i) g.c.d. $(n, \sigma(n))=n$ or (ii) g.c.d. $(n, \sigma(n)) \neq n$.(i) implies that $\sigma(n)=k n$, where $k$ is a positive integer. In fact if $\sigma(n)=2 n$, then the numbers are called perfect numbers. Ore [9] proved that every perfect number is harmonic. But the converse is not true. For example, 140 is not perfect, but $H(140)=5$. Ore [4] conjectured that all harmonic numbers other than 1 must be even.

In the present paper, we focus on those harmonic numbers which satisfies the property (i). Some examples of this kind of numbers are $6,28,496,672,8128,30240, \ldots \ldots$. In section 2 , some properties of the numbers of the form $p^{m} q$ where $\mathrm{p}, \mathrm{q}$ are any prime numbers have been discussed. In section 3 , the type of numbers have been extended to the form $2^{k} p^{m} q$. In section 4 , the numbers have been further extended to the form $2^{k} p_{1} p_{2} \ldots \ldots p_{m}$. In fact in this section a search technique have been obtained to get the numbers of the form g.c.d. $(n, \sigma(n))=n$, where $n=2^{k} p_{1} p_{2} \ldots \ldots p_{m}$.

II. Some properties of harmonic numbers having g.c.d. $(n, \sigma(n))=n$ of the form $p^{m} q$ Theorem 2.1 The only harmonic divisor number of the form $p q$, where $p$ and $q$ are prime numbers and g.c.d. $(n, \sigma(n))=n$, is 6 .

Proof: Let $n=p q$, where $p, q$ are two prime numbers. Now $\sigma(n)=1+p+q+p q$. If $\mathrm{n}$ divides $\sigma(\mathrm{n})$ completely then $1+p+q+p q=k p q$, for some number $\mathrm{k}$. Since $\mathrm{n}$ is a harmonic divisor number so $k \mid \tau(n)=4$. So the possible values of $\mathrm{k}$ are $1,2,4$. Since $1+p+q \neq 0$ therefore $k \neq 1$. The other possible equations are $1+p+q=p q$ or $1+p+q=3 p q$. The possible values of $p$ are $\frac{1+q}{q-1}, \frac{1+q}{3 q-1}$. Now $1+q>$ $3 q-1=>2 q<2$, which is not possible. Hence the only possible solutions are 2,3. Therefore 6 is the only harmonic divisor number of the form $p q$.

Theorem 2.2 The only harmonic divisor number of the form $p^{2} q$, where $p$ and $q$ are prime numbers such that g.c.d. $(n, \sigma(n))=n$ is 28 . 
Proof: Here $\sigma(n)=1+p+p^{2}+q+p q+p^{2} q$ and $(n)=6$. To satisfy the condition $n \mid \sigma(n)$ we have $q=\frac{1+p+p^{2}}{-1-p-p^{2}+k p^{2}}$, where $\sigma(n)=n k$. The possible values of $\mathrm{k}$ are $1,2,3,6$. Clearly $\mathrm{k}$ cannot be 1 .As $\sigma(n)=n k$ implies $\sigma(n)=n$ i.e. $1+p+p^{2}+q+p q+p^{2} q=p^{2} q$, i.e. $1+p+p^{2}+q+p q=0$, which is not possible as the LHS is greater than 0 . Let $\quad k p^{2}-\left(1+p+p^{2}\right)=t$ and

$$
1+p+p^{2}=q t
$$

Where $\mathrm{t}$ is some positive integer. Adding (1) and (2), $k p^{2}=(q+1) t$. Firstly, we consider that both $p$ and $q$ are odd primes. From (2) we have $q$ and $t$ are odd numbers as $q t$ must be odd, which is because, $1+p+p^{2}$ is odd. Now from (1) $k p^{2}$ must be even as the RHS is odd. As $p$ is an odd prime, $k$ must be even. Hence the possible values of $k$ are 2,6

Case (i). $\quad k=2$

We have

$$
\begin{aligned}
& k p^{2}=(q+1) t \\
\Rightarrow & 2 p^{2}=(q+1) t \\
\Rightarrow & 2 \cdot p \cdot p=(q+1) t
\end{aligned}
$$

Then the possible equations are $q+1=2 p^{2}, t=1$ or $q+1=2 p, t=p$

If $q+1=2 p^{2}, t=1$, then equation (2) implies $1+p+p^{2}=\left(2 p^{2}-1\right) \cdot 1 \Rightarrow p^{2}-p-2=0$, which has no prime solutions. Similarly, if $1+p+p^{2}=(2 p-1) p$ then $p^{2}-2 p-1=0$, which does not give any prime values of $\mathrm{p}$. Therefore $k$ cannot be 2 .

Case (ii). $\quad k=6$

$$
\begin{array}{ll}
\text { We have } & k p^{2}=(q+1) t \\
& \Rightarrow 6 p^{2}=(q+1) t \\
& \Rightarrow 2 \cdot 3 \cdot p \cdot p=(q+1) t
\end{array}
$$

The possible values of $(q, t)$ are $\left(2,3 p^{2}\right),\left(6, p^{2}\right),(2 p, 3 p),\left(2 p^{2}, 3\right),(6 p, p),\left(6 p^{2}, 1\right)$. Clearly any of the above pair does not satisfy the equation (2) for odd prime $p$. Next we assume that $p$ is an even prime. From equation (2), we have $1+p+p^{2}=q t \Rightarrow q t=7$. Since $q$ is prime the only possibility is $q=7$. Hence the number becomes $n=2^{2}$.7. Also we have $\tau(n)=6$ and $\sigma(n)=56$. Therefore $\sigma(n)=2 n$ and $H(n)=3$. Similarly if we consider $q$ is even then equation (2) is not satisfied as the LHS is odd and the RHS is even. Hence the theorem.

In fact the above theorem may be generalised to some extent and may be stated as follows:

Theorem 2.3 If $n$ is a harmonic number of the form $p^{m} q$, where $p, q$ are prime numbers and $m$ is a positive integer such that $g . c . d .(n, \sigma(n))=n$ then $H(n)=\frac{\tau(n)}{2}$

Proof: Since $n \mid \sigma(n)$ and $n=p^{m} q$, therefore $q$ can be expressed as

Let

$$
q=\frac{\sum_{i=0}^{i=m} p^{i}}{k p^{m}-\sum_{i=0}^{i=m} p^{i}}
$$

$$
k p^{m}-\sum_{i=0}^{i=m} p^{i}=t
$$

$\sum_{i=0}^{i=m} p^{i}=q t$

Where $t$ is a particular positive integer .Adding (3) and (4), we have $k p^{m}=(q+1) t$

Again

So $k$ is either 1 or 2 .

$$
\begin{aligned}
& q=\frac{\sum_{i=0}^{i=m} p^{i}}{k p^{m}-\sum_{i=0}^{i=m} p^{i}}>1 \\
\Rightarrow & 2 \sum_{i=0}^{i=0} p^{i}>k p^{m} \\
\Rightarrow & 2 \frac{p^{m+1}-1}{p-1}>k p^{m} \\
\Rightarrow & k<\frac{2\left(p^{m+1}-1\right)}{p^{m}(p-1)} \\
\Rightarrow & k<\frac{2\left(p-\frac{1}{p^{m}}\right)}{(p-1)} \\
\Rightarrow & k<2\left\{\frac{p}{p-1}-\frac{1}{p^{m}(p-1)}\right\} \\
\Rightarrow & k \leq 2
\end{aligned}
$$

If $k=1$, then from equation (3),we have

$$
\begin{aligned}
& p^{m}-\sum_{i=0}^{i=m} p^{i}=t \\
& p^{m}-\sum_{i=0}^{i=m-1} p^{i}-p^{m}=t \\
& -\sum_{i=0}^{i=m-1} p^{i}=t
\end{aligned}
$$

Which is not possible as the LHS is negative and RHS is positive. Therefore $k$ must be 2. i.e. $H(n)=\frac{\tau(n)}{2}$. Hence the theorem is proved. 
In fact, it has been observed by Pomerance [10] and Callan [7] that the only harmonic numbers of the form $p^{a} q^{b}$ are perfect numbers.

Theorem 2.4 There is no perfect number of the form $p^{3} q$, where $p, q$ are odd primes.

Proof: Let $n=p^{3} q$ then $\sigma(n)=\left(1+p+p^{2}+p^{3}\right)(1+q)$. Since we are considering the perfect number ,therefore $\sigma(n)=2 n$. That is $\left(1+p+p^{2}+p^{3}\right)(1+q)=2 p^{3} q$.

Or $\quad\left(\frac{1+p+p^{2}+p^{3}}{p^{3}}\right)\left(\frac{1+q}{q}\right)=2$

Suppose if possible $p \mid\left(1+p+p^{2}+p^{3}\right)$. Obviously $p \mid\left(p+p^{2}+p^{3}\right)$. Therefore, we have $p \mid((1+$ $p+p 2+p 3-p+p 2+p 3)$. i.e. $p / 1$, which is not possible. Hence $p \nmid(1+p+p 2+p 3)$. Therefore the only possibility is $p^{3} \mid(1+q)$ and $q \mid\left(1+p+p^{2}+p^{3}\right)$. 2 being a prime number the only possibilities are (i) $2 p^{3}=1+q$ and $q=1+p+p^{2}+p^{3}$. But second one is not possible as the LHS is odd and the RHS is even. and (ii) $2 q=1+$ $p+p^{2}+p^{3}$ and $p^{3}=1+q$.Again the second one is not possible as the LHS is odd and the RHS is even .Hence the Result.

The above theorem may be generalized as:

Theorem 2.5 There is no perfect number of the form $p^{m} q$, where $p$ and $q$ are odd primes and $m$ is an odd numbers.

Proof: Let $n=p^{m} q$ then $\sigma(n)=\left(\sum_{i=0}^{i=m} p^{i}\right)(1+q)$. Since we considering the perfect number therefore $\sigma(n)=2 n$. That is $\left(\sum_{i=0}^{i=m} p^{i}\right)(1+q)=2 p^{m} q$

Or $\quad\left(\frac{\left(\sum_{i=0}^{i=m} p^{i}\right)(1+q)}{p^{m} q}\right)=2$

Suppose, if possible $p \mid\left(\sum_{i=0}^{i=m} p^{i}\right)$. Obviously $p \mathrm{I}\left(\sum_{i=1}^{i=m} p^{i}\right)$. Therefore, we have $p \mid\left(\sum_{i=0}^{i=m} p^{i}\right)-$ $\left(\sum_{i=1}^{i=m} p^{i}\right)$. i.e. $p \mid 1$, which is not possible. Hence $p \nmid \cdot\left(\sum_{i=0}^{i=m} p^{i}\right)$. Therefore the only possibility is $p^{m} \mid 1+q$ and $q \mathrm{I}\left(\sum_{i=0}^{i=m} p^{i}\right) .2$ being a prime number the possibilities are (i) $2 p^{m}=1+q$ and $q=\sum_{i=0}^{i=m} p^{i}$. But second one is not possible, as the LHS is odd and the RHS is even. (ii) $2 q=\sum_{i=0}^{i=m} p^{i}$ and $p^{m}=1+q$, again second one is not possible as the LHS is odd and the RHS is even and so the result. Theorem $\mathbf{2 . 4}$ is a particular case when $\mathrm{m}=3$.

We now state that "There is no harmonic number of the form $\boldsymbol{p}^{\boldsymbol{m}} \boldsymbol{q}$, where $\mathbf{p}$ and $q$ are odd primes and $m$ is an odd number such that $g . c . d .(n, \sigma(n))=n$."

\section{Some properties of the harmonic numbers having $g . c . d .(n, \sigma(n))=n$ of the form}

$$
2^{k} p^{m} q
$$

In this section we are trying to find the existence of some harmonic numbers of the form $2^{k} p^{m}$, for small values of $\mathrm{m}$.

Theorem 3.1 Let the number be of the form $n=2^{k} p$ and let g.c.d. $(n, \sigma(n))=n$, then there exists at least one $n$ such that $H(n)=\frac{\tau(n)}{2}$

Proof: If $n=2^{k} p$ then $\sigma(n)=\sum_{i=0}^{i=k} 2^{i}(1+p)$. Since $\sigma(n)=n k_{1}$, for some number $\mathrm{k}_{1}$. We have

$$
\begin{gathered}
n k_{1}=\sum_{i=0}^{i=k} 2^{i}(1+p) \\
2^{k} p k_{1}=\sum_{i=0}^{i=k} 2^{i}(1+p) \\
k_{1}=\frac{1+p}{p}\left(\frac{2^{k+1}-1}{2^{k}}\right)
\end{gathered}
$$

Let $H(n)$ be the harmonic mean divisors of $\mathrm{n}$. Then $k_{1} H(n)=\tau(n)$

Hence

$$
\begin{gathered}
\tau(n)=H(n) \frac{1+p}{p}\left(\frac{2^{k+1}-1}{2^{k}}\right) \\
(1+k) 2=H(n) \frac{1+p}{p}\left(\frac{2^{k+1}-1}{2^{k}}\right) \\
(1+k)=H(n) \frac{1+p}{p}\left(\frac{2^{k+1}-1}{2^{k+1}}\right) \ldots
\end{gathered}
$$

We observed that if $p=2^{k+1}-1$ then $H(n)=\frac{\tau(n)}{2}$.

With a small program in MATHEMATICA software it can be easily seen that some of the values of $\mathrm{k}+1$, for which $p=2^{k+1}-1 \quad$ is a prime number are $\{2,3,5,7,13,17,19,31,61,89,107,127,521,607,1279,2203,2281,3217,4253,4423,9689,9941\}$. However it has been observed that if $p=2^{k+1}-1$ is a prime number then $k+1$ itself must be a prime number. These 
numbers are called Mersene primes and the numbers of the form $2^{k}\left(2^{k+1}-1\right)$ are called perfect numbers. So far there are 48 Mersene primes .

Theorem 3.2 There does not exist any harmonic number of the form $n=2^{k} p^{2}$ for which g.c.d. $(n, \sigma(n))=n$, where $k$ is a positive integer and $p$ is an odd prime.

Proof: For $n=2^{k} p^{2}$, we have $\sigma(n)=\left(2^{k+1}-1\right)\left(1+p+p^{2}\right)$. Then

$$
\frac{\sigma(n)}{n}=\frac{\left(2^{k+1}-1\right)\left(1+p+p^{2}\right)}{2^{k} p^{2}}
$$

Now $2^{k} \nmid\left(2^{k+1}-1\right)$ and $2^{k} \nmid\left(1+p+p^{2}\right)$, as $p$ is an odd prime. Hence $1+p+p^{2}$ becomes odd. Hence the theorem.

The above theorem is possible as $1+p+p^{2}$ becomes odd. So this will happen till $\sum p^{i}$ is odd for some values of $i$, in fact if $i$ is even. So we can state the following result:

Theorem 3.3 There does not exists any harmonic number of the form $2^{k} p^{2 x}$ of which g.c.d. $(n, \sigma(n))=$ $n$ where $k, x$ are positive integers and $p$ is an odd prime.

Theorem 3.4 There is no harmonic numbers up to $10^{76}$ of the form $2^{k} p^{3}$, where $k$ is a positive integer and $p$ is odd prime.

Proof: Let $n=2^{k} p^{3}$. Then $\sigma(n)=\left(2^{k+1}-1\right)\left(1+p+p^{2}+p^{3}\right)$ and $\tau(n)=4(k+1)$. As g.c.d. $(n, \sigma(n))=n$ we may assume $\sigma(n)=n k_{1}$ where $k_{1}$ is an integer.

Then $\quad k_{1}=\frac{\left(2^{k+1}-1\right)\left(1+p+p^{2}+p^{3}\right)}{2^{k} p^{3}}$ is also an integer.

Now $p \mid\left(p+p^{2}+p^{3}\right)$, if we assume $p \mid\left(1+p+p^{2}+p^{3}\right)$ then $p \mid\left(1+p+p^{2}+p^{3}\right)-\left(p+p^{2}+p^{3}\right)$ i.e. $p \mid 1$, which is not possible. Hence $p \nmid\left(1+p+p^{2}+p^{3}\right)$ i.e. $p^{3} \nmid\left(1+p+p^{2}+p^{3}\right)$. Therefore the only possibility is $p^{3} \mid\left(2^{k+1}-1\right)$. As . $2^{k} \nmid\left(2^{k+1}-1\right)$ so $2^{k} \mid\left(1+p+p^{2}+p^{3}\right)$.

Let

$$
x p^{3}=2^{k+1}-1 \text {. }
$$

And

$$
1+p+p^{2}+p^{3}=y 2^{k}
$$

for some positive integer $x, y$. It is clear that for large value of $2^{k+1}-1$, if $p$ is comparatively very small then the equation (9) is not satisfied for $y \geq 1$. We have searched the numbers of the form $2^{k+1}-1$ for $1 \leq k \leq$ 251 in MATHEMATICA for which the value of $p$ has been found to be very very small. Hence all these numbers does not satisfy the second equation. Hence there is no number of the form $2^{k} p^{3}$ for $k \leq 251$.i.e. there is no harmonic numbers up to $10^{76}$ of the form $2^{k} p^{3}$.

Theorem 3.5 Let the number be of the form $n=2^{k} p q$ and let g.c.d. $(n, \sigma(n))=n$ then there exists no $n$ such that $H(n)=\frac{\tau(n)}{2^{x}}, x \geq 1$

Proof: For $n=2^{k} p q$, we have $\sigma(n)=\left(2^{k+1}-1\right)(1+p)(1+q)$ and $\tau(n)=4(k+1)$. Let $\sigma(n)=n k_{1}$ where $k_{1}$ a positive integer. Therefore

as $k_{1} H(n)=\tau(n)$

$$
k_{1}=\frac{\left(2^{k+1}-1\right)(1+p)(1+q)}{2^{k} p q}
$$

Or

For $H(n)=\frac{\tau(n)}{2}$,

$$
\begin{aligned}
& (1+k) 4=H(n)\left(\frac{1+p}{p}\right)\left(\frac{1+q}{q}\right)\left(\frac{2^{k+1}-1}{2^{k}}\right) \\
& (1+k) 2=H(n)\left(\frac{1+p}{p}\right)\left(\frac{1+q}{q}\right)\left(\frac{2^{k+1}-1}{2^{k+1}}\right)
\end{aligned}
$$

$$
\begin{array}{cr} 
& \left(\frac{1+p}{p}\right)\left(\frac{1+q}{q}\right)\left(\frac{2^{k+1}-1}{2^{k+1}}\right)=1 \\
\text { Or } & \left(2^{k+1}-1\right)(1+p+q)=p q \ldots \ldots \ldots \ldots \ldots \ldots \ldots
\end{array}
$$

If $p=x\left(2^{k+1}-1\right)$ for $x>1$ then $q$ is a composite number. Same is true for $q$.Hence the only possibility is $p$ or $q$ must be of the form $2^{k+1}-1$. But if $p=2^{k+1}-1$ then from the equation (10), we have $1+p+q=q$ i.e. $p=-1$ is not possible. Same is true for $\mathrm{q}$ also. Thus $H(n)=\frac{\tau(n)}{2}$ is not possible for all $p$ and $q$ odd primes. Now we can consider the case of $H(n)=\frac{\tau(n)}{2^{x}}, x>1$. Then we have $\frac{\sigma(n)}{2^{x} n}=1$. This implies that 


$$
\begin{gathered}
\left(\frac{1+p}{p}\right)\left(\frac{1+q}{q}\right)\left(\frac{2^{k+1}-1}{2^{k+x}}\right)=1 \\
\left(\frac{1+p+q}{p q}+1\right)\left(\frac{2^{k+1}-1}{2^{k+x}}\right)=1 \\
\left(\frac{1+p+q}{p q}\right)\left(\frac{2^{k+1}-1}{2^{k+x}}\right)=1-\left(\frac{2^{k+1}-1}{2^{k+x}}\right) \\
(1+p+q)\left(2^{k+1}-1\right)=\left(2^{k+x}-2^{k+1}+1\right) p q \\
(1+p+q)\left(2^{k+1}-1\right)=\left\{2^{k+1}\left(2^{x-1}-1\right)+1\right\} p q .
\end{gathered}
$$

For $x \geq 2,\left\{2^{k+1}\left(2^{x-1}-1\right)+1\right\}>\left(2^{k+1}-1\right)$. Also $p q>(1+p+q)$. Hence the RHS of the equation (11) is greater than the LHS, which is not possible. Hence the theorem.

\section{Theorem 3.6 If a number is of the form $n=2^{k} p^{2} q$ with g.c.d. $(n, \sigma(n))=n$ and $H(n)=\frac{\tau(n)}{2}$, then $n$ does not exist.}

Proof: For $n=2^{k} p^{2} q$ we have $\sigma(n)=2^{k+1}\left(1+p+p^{2}\right)(1+q)$ and $\tau(n)=3(k+1) 2$. Let $\sigma(n)=n k_{1}$ where $k_{1}$ is a positive integer. Then we have

$$
\left(2^{k+1}-1\right)\left(1+p+p^{2}\right)(1+q)=2^{k} p^{2} q k_{1}
$$

Or

$$
\left(\frac{2^{k+1}-1}{2^{k}}\right)\left(\frac{1+p+p^{2}}{p^{2}}\right)\left(\frac{1+q}{q}\right)=k_{1}
$$

Again

$$
H(n)=\frac{n \tau(n)}{\sigma(n)}=\frac{\tau(n)}{k_{1}}
$$

Or

$$
\begin{gathered}
\left(\frac{2^{k+1}-1}{2^{k}}\right)\left(\frac{1+p+p^{2}}{p^{2}}\right)\left(\frac{1+q}{q}\right)=\frac{\tau(n)}{H(n)} \\
H(n)\left(\frac{2^{k+1}-1}{2^{k}}\right)\left(\frac{1+p+p^{2}}{p^{2}}\right)\left(\frac{1+q}{q}\right)=3(1+k) 2 \\
H(n)\left(\frac{2^{k+1}-1}{2^{k+1}}\right)\left(\frac{1+p+p^{2}}{p^{2}}\right)\left(\frac{1+q}{q}\right)=3(1+k) . \ldots .
\end{gathered}
$$

A necessary condition that $H(n)\left(\frac{2^{k+1}-1}{2^{k+1}}\right)\left(\frac{1+p+p^{2}}{p^{2}}\right)\left(\frac{1+q}{q}\right)$ is an integer.

Suppose we want to make $H(n)=3(1+k)$

Then $\quad\left(\frac{2^{k+1}-1}{2^{k+1}}\right)\left(\frac{1+p+p^{2}}{p^{2}}\right)\left(\frac{1+q}{q}\right)=1$

As $2^{k+1}-1,1+p+p^{2}$ are odd numbers so only possibility is $2^{k+1} \mid 1+q$.

Let $q=x 2^{n}-1$, where $n \geq k+1$

If $n>k+1$ then the expression (13) becomes

$$
\left(2^{k+1}-1\right)\left(\frac{1+p+p^{2}}{p^{2}}\right)\left(\frac{x 2^{y}}{q}\right)=1 \text { for some } y \geq 0
$$

As $p$ and $q$ are odd primes the only possibility is $y=0$ i.e. $n=k+1$

Hence the expression (14) becomes

$$
\left(2^{k+1}-1\right)\left(\frac{1+p+p^{2}}{p^{2}}\right)\left(\frac{x}{q}\right)=1
$$

For $x \geq 1, q$ does not divide $2^{k+1}-1$, hence the only possibility is $q \mid\left(1+p+p^{2}\right)$

Let $q t=1+p+p^{2}$ for some positive integer $t$, then the expression (15) becomes

$$
\left(2^{k+1}-1\right) \frac{t x}{p^{2}}=1
$$

As $\quad x>1$, and $2^{k+1}-1>1$, the only possibility is $t=1$ and $\quad x=2^{k+1}-1=p$

Now $\quad q=1+p+p^{2}=>x 2^{k+1}-1=1+p+p^{2}$

$$
=>p 2^{k+1}-p=2+p^{2}=>p\left(2^{k+1}-1\right)=2+p^{2}
$$

i.e. $p^{2}=2+p^{2}$, which is not possible.

Now, we consider $x=1$. then $q=2^{k+1}-1$. Therefore the equation (16) becomes $\left(2^{k+1}-1\right) \frac{t}{p^{2}}=1$

If $t=1$ then $p^{2}=2^{k+1}-1=q$. Therefore $q t=1+p+p^{2}$ implies $q=1+p+p^{2}$, which gives $p=-1$ ,which is not possible.

Next we consider that $t>1$. Again there are two possibilities, $t=p$ and $t=p^{2}$. If $t=p^{2}$ then $2^{k+1}-1=1$. This implies that $k=0$ which is not acceptable .Lastly we have $t=p$. Then $2^{k+1}-1=p$ i.e. $p=q$. So $q t=1+p+p^{2}$ implies $p^{2}=1+p+p^{2}$ i.e. $p=-1$, not possible . Thus there exists no number of the form $n=2^{k} p^{2} q$ such that $\mathrm{H}(\mathrm{n})=3(k+1)=\frac{\tau(n)}{2}$. The above theorem may be generalized on the power of of $\mathrm{p}$ and can be written as follows: 
Theorem 3.7 Let the number be of the form $n=2^{k} p^{2 x} q$ where $x$ is any positive integer and $p, q$ are odd primes. Let g.c.d. $(n, \sigma(n))=n$ and $H(n)=\frac{\tau(n)}{2}$, then $n$ does not exist.

Proof: If $H(n)=\frac{\tau(n)}{2}$ then we have

$$
\left(\frac{2^{k+1}-1}{2^{k+1}}\right)\left(\frac{\sum_{i=0}^{i=2 x} p^{i}}{p^{2 x}}\right)\left(\frac{1+q}{q}\right)=1
$$

As $2^{k+1}-1$ and $\sum_{i=0}^{i=2 x} p^{i}$ are odd numbers, so $2^{k+1} \mid 1+q$.

Let $q=x_{1} 2^{n}-1$, where $n \geq k+1$

If $n>k+1$ then the expression (17) becomes

$$
\left(2^{k+1}-1\right)\left(\frac{\sum_{i=0}^{i=2 x} p^{i}}{p^{2 x}}\right) \frac{x 2^{y}}{q}=1 \text {, for some } y \geq 1
$$

For $x \geq 1, q$ does not divide $2^{k+1}-1$, so the only possibility is $q \mid \sum_{i=0}^{i=2 x} p^{i}$. Let $q t=\sum_{i=0}^{i=2 x} p^{i}$, then the equation (18) becomes

The only possibility is $1,2,3$

Now

$$
\left(2^{k+1}-1\right) \frac{t x_{1}}{p^{2 x}}=1
$$

$$
2^{k+1}-1=p^{y_{1}}, t=p^{y_{2}}, \quad x_{1}=p^{y_{3}}, \text { where } y_{1}+y_{2}+y_{3}=2 x \text { and } y_{i} \geq 0, i=
$$

$$
\begin{aligned}
& \begin{aligned}
q t & =\sum_{i=0}^{i=2 x} p^{i}
\end{aligned} \\
& =>\left(x_{1} 2^{k+1}-1\right) p^{y_{2}}=\sum_{i=0}^{i=2 x} p^{i} \\
& =>p^{y_{2}+y_{3}} 2^{k+1}-p^{y_{2}+y_{3}}=1+p^{y_{2}}+\sum_{i=1, i \neq y_{2}+y_{3}}^{i=2 x} p^{i} \\
& =>p^{y_{2}+y_{3}}\left(2^{k+1}-1\right)=1+p^{y_{2}}+\sum_{i=1, i \neq y_{2}+y_{3}}^{i=2 x} p^{i} \\
& =>0=1+p^{y_{2}}+\sum_{i=1, i \neq y_{2}+y_{3}}^{i=2 x-1} p^{i}
\end{aligned}
$$

This is not possible. Hence the theorem.

In theorem 3.6 we have seen that there is no harmonic number of the form $n=2^{k} p^{2} q$ such that $n$ completely divides $\sigma(n)$.However there are some harmonic numbers of this form such that $n$ does not divide completely $\sigma(n)$. Let $q=2^{k+1}-1$. Then, we have

$$
\begin{array}{ll} 
& \frac{H(n) \sigma(n)}{n}=\tau(n) \\
\text { i.e. } & H(n)\left(\frac{2^{k+1}-1}{2^{k+1}}\right)\left(\frac{1+p+p^{2}}{p^{2}}\right)\left(\frac{1+q}{q}\right)=3(1+k) \\
\text { i.e. } & H(n)\left(\frac{1+p+p^{2}}{p^{2}}\right)=3(1+k)
\end{array}
$$

As g.c.d. $\left(p^{2}, 1+p+p^{2}\right)=1$, the only possibility is $3(1+k)=y\left(1+p+p^{2}\right)$. Taking $y=3$ some of the numbers are $2^{12} 3^{2}\left(2^{13}-1\right), 2^{30} 5^{2}\left(2^{31}-1\right)$, taking $y=1$ some of the numbers are $2^{126} 19^{2}\left(2^{127}-1\right)$, $2^{60} 13^{2}\left(2^{61}-1\right), 2^{18} 7^{2}\left(2^{19}-1\right)$.

\section{Some properties of harmonic numbers having g.c.d. $(n, \sigma(n))=n$ of the form}

$$
2^{k} p_{1} p_{2} \ldots \ldots p_{m}
$$

Theorem 4.1 Let the number be of the form $n=2^{k} p_{1} p_{2} \ldots \ldots p_{m}$. Let one of them is $2^{k+1}-1$ then $\sigma(n) \neq k n$, where $k=2 x$, $x$ being odd and $p_{i}$ are odd prime, $i=1,2, \ldots, m$

Proof: We have $\sigma(n)=\left(2^{k+1}-1\right) \prod_{i=1}^{m}(1+p)_{i}$. Let $p_{1}=2^{k+1}-1$. If possible, let $\sigma(n)=2 x n$ where $x$ is an odd number. We have

$\mathrm{Or}$

$$
\begin{aligned}
& \frac{2^{k+1}-1}{2^{k+1}} \prod_{i=1}^{m} \frac{1+p_{i}}{p_{i}}=x \\
& \prod_{i=1}^{m} \frac{1+p_{i}}{p_{i}}=x
\end{aligned}
$$

This is not possible because the numerator part of LHS of (20) is even number and denominator part being odd the LHS becomes even. But the RHS is odd. This theorem can be generalized further to give the following theorem:

Theorem 4.2 Let the number be of the form $n=2^{k} p_{1} p_{2} \ldots \ldots . p_{m}$, where all $p$ 's are distinct odd primes, then $\sigma(n) \neq 2 n$.

Proof: Without loss of generality, we consider that $p_{1}<p_{2} \ldots \ldots \ldots \ldots \ldots p_{m}$.

Now

$$
\frac{\sigma(n)}{n}=\frac{2^{k+1}-1}{2^{k+1}} \prod_{i=1}^{m} \frac{1+p_{i}}{p_{i}}=k_{1}(\text { say })
$$

Where $k$ is a positive integer. Now $p_{m} \nmid\left(1+p_{i}\right), \forall i=1,2, \ldots \ldots, m$ as $p_{m}>p_{i}, \forall i=1,2, \ldots \ldots, m-1$. Therefore only possibility is $p_{m} \mid 2^{k+1}-1$. Let $2^{k+1}-1=r_{m} p_{m}, \quad r_{m} \geq 1$. 
So

$$
\begin{aligned}
\frac{\sigma(n)}{n} & =\frac{2^{k+1}-1}{2^{k+1}}\left(\frac{1+p_{m}}{p_{m}}\right) \prod_{i=1}^{m-1} \frac{1+p_{i}}{p_{i}} \\
& =\left(\frac{r_{m} p_{m}}{2^{k}}\right)\left(\frac{2^{k+1}-1+r_{m}}{r_{m} p_{m}}\right) \prod_{i=1}^{m-1} \frac{1+p_{i}}{p_{i}} \\
& =\frac{1}{2^{k}}\left(2^{k+1}-1+r_{m}\right)\left(\frac{1+p_{m-1}}{p_{m-1}}\right) \cdot \prod_{i=1}^{m-2} \frac{1+p_{i}}{p_{i}}
\end{aligned}
$$

Now $p_{m-11} \nmid\left(1+p_{i}\right), \forall i=1,2, \ldots ., m-1$ as $p_{m-1}>p_{i}, \forall i=1,2, \ldots . ., m-2$.

So the possibility is $p_{m-1} \mid 2^{k+1}-1+r_{m}$. Let $2^{k+1}-1+r_{m}=r_{m-1} p_{m-1}$, Then the equation (22) can be written as

$$
\frac{\sigma(n)}{n}=\frac{1}{2^{k}}\left(r_{m-1} p_{m-1}\right)\left(\frac{2^{k+1}-1+r_{m}+r_{m-1}}{r_{m-1} p_{m-1}}\right) \prod_{i=1}^{m-2} \quad \frac{1+p_{i}}{p_{i}}
$$

Continuing this process at the $j$ th stage, where the remaining primes are $p_{1}, p_{2} \ldots \ldots \ldots \ldots \ldots p_{j}$.

We have $\quad \frac{\sigma(n)}{n}=\frac{1}{2^{k}}\left(r_{j} p_{j}\right)\left(\frac{2^{k+1}-1+\sum_{i=j}^{m} r_{j}}{r_{j} p_{j}}\right) \prod_{i=1}^{j-1} \frac{1+p_{i}}{p_{i}}$

and $\quad r_{j-1} p_{j-1}=2^{k+1}-1+\sum_{i=j}^{m} r_{j}$. Therefore at the $\mathrm{m}$-th stage we have

$$
\frac{\sigma(n)}{n}=\frac{1}{2^{k}}\left(2^{k+1}-1+\sum_{i=1}^{m} r_{i}\right)
$$

If possible, let $\frac{\sigma(n)}{n}=2$, then the equation (23) becomes $\left(2^{k+1}-1+\sum_{i=1}^{m} r_{i}\right)=2^{k+1}$.So $\sum_{i=1}^{m} r_{i}=1$, which is not possible. Hence the result.

In fact the above theorem helps us to search numbers of the form $2^{k} p_{1} p_{2} \ldots \ldots . p_{m}$, where $p_{1}, p_{2}, \ldots \ldots, p_{m}$ are distinct odd primes and $p_{1<} p_{2}<, \ldots \ldots .<p_{m}$ (say), which are harmonic of course g.c.d. $(n, \sigma(n))=$ $n$.Following are the steps:

Step 1 . We chose the number $2^{k+1}-1$ and get its factor.It is desired to have the prime factors whose power is one .However if a prime factor is 2 or , say $q^{m}, m \geq 1$ occurs which is also a factor of $(k+1)$, then it may be allowed. We take the highest prime say $p_{m}$, as stated in the theorem, let $2^{k+1}-1=r_{m} p_{m}$

Step 2. We calculate the prime factor of $2^{k+1}-1+r_{m}$. In fact the prime factors of $2^{k+1}-1$ except $p_{m}$ will also be the prime factors of $2^{k+1}-1+r_{m}$. Hence the prime factors are never lost at any stage. Again it is desired to have the prime factors whose power is one. However if a prime factor is 2 or say $q^{m}, m \geq 1$ occurs which is also a factor of $(k+1)$, then it may be allowed.

Step 3. We repeat the above process and at the $\mathrm{j}$-th stage we consider the prime factors of $2^{k+1}-1+$ $\sum_{i=j}^{m} r_{i}$, again we choose the highest prime provided in the factorization all the prime factor other than prime factors of $(\mathrm{k}+1)$ are square free. We end the process when $2^{k+1}-1+\sum_{i=j}^{m} r_{i}$ has only prime factor 2.Harmonic mean $\mathrm{H}(\mathrm{n})$ of these numbers will be of the form $q_{1} q_{2} \ldots \ldots q_{i} 2^{j}$, for some $j$ where $q_{1}, q_{2}, \ldots ., q_{i}$ are some of the prime factors of $(\mathrm{k}+1)$. Since the searching process is dependent only on $k$, it takes less time to search the numbers of the said form out of a wide range of numbers. It has been observed that there is no numbers of the said form whose harmonic mean is $(k+1) 2^{x}$ for some $x$ up to $10^{100}$.

\section{References}

[1] D.Callan, Solution to Problem 6616, Amer.Math.Monthly 99 (1992),783-789,MR1542194

[2] G.L.Cohen, Numbers whose positive divisors have small integral harmonic mean. Mathematics of computation.Vol. 66, No.218,1997, PP 883-891.

[3] G.L.Cohen and R.M.Soril. Odd harmonic number exceed $10^{24}$, Mathematics of Computation, S 0025- 5718(10)02337-9

[4] T. Goto and S.Shobata. All numbers whose positive divisors have integral harmonic mean up to 300 , Mathematics of Computation, Vol. 73, No. 245, PP 473-491

[5] T. Goto and K.Okeya. All Harmonic numbers less than $10^{14}$, Japan J. Indust.Appl. Math.24(2007), 275-288

[6] M.Gracia. On numbers with integral harmonic mean, Amer. Math. Monthly, 61(1954), 89-96. MR 15:506d

[7] R.K.Guy. Unsolved Problems in Number Theory, 3rd edition. Springer-Verlag, New York, 2004.

[8] G.H.Hardy and E.M.Wright. An Introduction to the Theory of Numbers, fourth edition, Oxford (1962). MR0067125(16:673c)

[9] O.Ore. On the averages of the divisors of a number, Amer.Math.Monthly, 55 (1948), PP 615-619

[10] C. Pomerance. On a problem of Ore: harmonic numbers, unpublished manuscript (1973) see abstract *709-A5, Notice Amer.Math.soc. 20(1973), a-648

[11] Solution to Problem E3445, Amer. Math.Monthly 99(1992),795

[12] J.Spiess. 1990, Some identities involving harmonic numbers, Math.Comp.55,839-863. 